\title{
Stroke rehabilitation: long-term rehabilitation after stroke
}

\author{
Authors: Katharina Dworzynski, ${ }^{A}$ Gill Ritchie ${ }^{B}$ and E Diane Playford ${ }^{C}$
}

Many stroke survivors, despite improvements in mortality and morbidity, remain dependent on others for everyday activities. People with stroke need access to effective specialist multidisciplinary rehabilitation services that are organised and integrated within the whole system of health and social care. They also commonly come under the care of generalists in various clinical contexts, including intercurrent illness. This Clinical Medicine Concise Guideline abstracts key recommendations from the National Institute for Health and Care Excellence Clinical Guideline 162 on stroke rehabilitation of particular relevance to clinicians in general medicine, to inform their front-line practice and promote liaison and collaboration with the specialist service.

KEYWORDS: Stroke, rehabilitation, multidisciplinary

\section{Introduction}

Each year approximately 110,000 people in England, 11,000 in Wales and 4,000 in Northern Ireland have a first or recurrent stroke. There are more than 900,000 individuals who have had a stroke living in England alone. ${ }^{1}$ Most people survive a first stroke, but often have significant morbidity. The development of stroke units following the Stroke Unit Trialists' Collaboration meta-analysis, ${ }^{2}$ and further reorganisation of services following the advent of thrombolysis have significantly improved mortality and morbidity. ${ }^{1}$ Over $30 \%$ of people, however, have persisting disability and corresponding service needs in the long term.

The age-associated incidence of stroke predicts an increasing need with population ageing, and a corresponding need for skilled management of stroke morbidity in the context of prevalent comorbidity within the older population. Clinicians in general medical specialities will increasingly encounter stroke sufferers beyond the acute stage in their routine practice, especially in the context of intercurrent illness which may itself impact adversely on any stroke-related disability.

Authors: A senior research fellow and guideline lead Royal College of Obstetricians and Gynaecologists, London, UK; ${ }^{B}$ associate director, National Clinical Guideline Centre, Royal College of

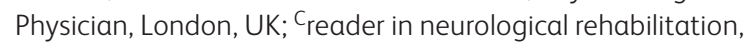
UCL Institute of Neurology, London, UK
Understanding specific stroke-related deficits, the broader consequences for independent living, and the measures and services recommended for their management is therefore an integral element of general medicine, and key to effective liaison with specialist stroke rehabilitation services.

A stroke rehabilitation service comprises a core multidisciplinary team who have the knowledge, skills and experience to work in partnership with people with stroke and their families and carers. Key aspects include multidisciplinary assessment, identification and measurement of functional difficulties, treatment planning through goal setting, delivery of interventions (either to effect change or support the individual in managing persisting change), and evaluation of effectiveness.

National Institute for Health and Care Excellence (NICE) Clinical Guideline 162 (CG162), ${ }^{3}$ which should be read in association with (i) $\mathrm{CG}^{4} 8^{4}$ and (ii) the Intercollegiate Stroke Working Party National clinical guideline for stroke, ${ }^{5}$ deals with interventions in stroke rehabilitation care against the available evidence. Rehabilitation starts in the acute setting, but continues into the community and informs long-term living with stroke. The CG162 scope is explicit that not every domain of the discipline is exhaustively covered. This reflects partly its enormity (there is a potential case for separate freestanding guidelines on some subtopics) but also the very limited evidence in certain areas.

This Clinical Medicine Concise Guideline abstracts elements of CG162 important for an understanding of stroke rehabilitation, with emphasis on the context of general medicine - front-line assessment, planning and delivery of care, and liaison with the specialist service. These are presented under the main CG162 subheadings.

1. Organising health and social care for those needing rehabilitation after stroke

2. Planning and delivering stroke rehabilitation - detection, assessment and rehabilitation of individual functions, including cognitive functioning; emotional functioning;

The National Institute of Health and Care Excellence (NICE) has accredited the process used by the Royal College of Physicians to produce the concise clinical guidelines published in Clinical Medicine with effect February 2010 to March 2018 (abstracted guidance) and July 2013 to July 2018 (de novo guidance). More information on accreditation can be viewed at: www.nice.org.uk/about/what-we-do/accreditation. 
vision, swallowing, communication and movement, as well as self care and employment.

3. Providing support and information

4. Long-term health and social support

Recommendations abstracted verbatim from the guideline are highlighted (minor stylistic changes and/or repositioning only where necessary to minimise repetition and provide logical progression for this concise review).

\section{Organisation of health and social care}

The complexity of stroke - specific impairments and their consequences for reduction in physical ability, occupation and lifestyle - poses major challenges for coordination, communication and the organisation of intervention and support. Effective stroke rehabilitation requires such challenges to be overcome, (i) by means of organised, multidisciplinary practice in the hospital and/or community setting and (ii) by bridging gaps between hospital and community-based practice through continued, shared responsibility and accountability.

\section{Stroke units}

(i) People with disability after stroke should receive rehabilitation in a dedicated stroke inpatient unit and subsequently from a specialist stroke team within the community.

\section{The core multidisciplinary stroke team}

(ii) A core multidisciplinary stroke rehabilitation team should comprise the following professionals with expertise in stroke rehabilitation: consultant physicians; nurses, physiotherapists, occupational therapists, speech and language therapists, clinical psychologists, rehabilitation assistants and social workers.

\section{Health and social care interface}

(iii) Health and social care professionals should work collaboratively to ensure a social care assessment is carried out promptly, where needed, before transfer from hospital to the community. The assessment should:

$>$ identify any ongoing needs of the person and their family or carer, for example, access to benefits, care needs, housing, community participation, return to work, transport and access to voluntary services

> be documented and all needs recorded in the person's health and social care plan, with a copy provided to the person with stroke.

\section{Transfer of care from hospital to community}

Rehabilitation can take place either in hospital or at home. There are potential advantages to rehabilitation at home, including interventions targeted more accurately at need within that environment, enhanced wellbeing and mood for patients and carers, and greater cost-effectiveness. ${ }^{3}$ There are also potential disadvantages. High intensity therapy may be more difficult to organise in a community setting.
Free and effective communication and clear lines of accountability between hospital- and community-based services are therefore essential. Standard operating procedures and local protocols are required.

(iv) Before transfer of care from hospital to home for people with stroke, establish that they have a safe and enabling home environment:

$>$ check that appropriate equipment and adaptations have been provided and that carers are supported to facilitate independence

> undertake a home visit with them unless their abilities and needs can be identified in other ways, for example, by demonstrating independence in all self-care activities, including meal preparation, while in the rehabilitation unit.

Early supported discharge is a service which allows transfer of continued rehabilitation at the same level of intensity and expertise from an inpatient to a primary care setting.

(v) Offer early supported discharge to those who are able to transfer from bed to chair independently or with assistance, as long as a safe and secure environment can be provided.

(vi) Hospitals should have systems in place to ensure that:

$>$ people after stroke and their families and carers (as appropriate): (1) are involved in planning for transfer of care, and carers receive training in care (for example, in moving and handling and helping with dressing) and (2) feel adequately informed, prepared and supported

$>$ GPs and other appropriate people are informed before transfer of care

$>$ an agreed health and social care plan is in place, and the person knows who to contact if difficulties arise

$>$ appropriate equipment (including specialist seating and a wheelchair if needed) is in place at the person's residence, regardless of setting.

(vii) Documentation should be individualised, and should include the following information as a minimum:

$>$ basic demographics, including contact details and next of kin

$>$ diagnosis and relevant medical information

$>$ list of current medications, including allergies

$>$ standard screening assessments

$>$ the person's rehabilitation goals

$>$ multidisciplinary progress notes

$>$ a key contact from the stroke rehabilitation team (including their contact details) to coordinate the person's health and social care needs

> discharge planning information (including accommodation needs, aids and adaptations)

$>$ joint health and social care plans, if developed

$>$ follow-up appointments.

\section{Planning and delivering stroke rehabilitation}

In addition to a full medical review, effective rehabilitation requires accurate identification and assessment of the consequences of stroke and a corresponding programme 
of appropriate intensity, tailored and planned to individual needs. It should take into account previous functional abilities, any impairment of psychological (cognitive, emotional and communication) and physical function (including pain), any activity and participation limitations, and finally environmental factors (social, physical and cultural).

\section{Screening and assessment}

The generalist should be particularly aware of the following aspects of stroke-related need and ensure they are systematically addressed in tandem with care planning and liaison with the stroke team.

(viii) On admission to hospital, to ensure the immediate safety and comfort of the person with stroke, screen them for the following and, if problems are identified, start management as soon as possible:

$>$ orientation

$>$ positioning, moving and handling

$>$ swallowing

$>$ transfers (for example, from bed to chair)

$>$ pressure area risk

$>$ continence

$>$ communication, including the ability to understand and follow instructions and to convey needs and wishes

$>$ nutritional status and hydration (follow the recommendations in stroke (CG68) ${ }^{4}$ and nutrition support in adults (CG32). ${ }^{6}$

\section{Cognitive functioning}

(ix) Screen people after stroke for cognitive deficits. Where a cognitive deficit is identified, carry out a detailed assessment using valid, reliable and responsive tools before designing a treatment programme.

\section{Emotional functioning}

(x) Assess emotional functioning in the context of cognitive difficulties in people after stroke. Any intervention chosen should take into consideration the type or complexity of the person's neuropsychological presentation and relevant personal history.

\section{Setting goals for rehabilitation}

Consensus, localised effective interdisciplinary communication, involvement of family or carer, and continuity, are all essential to efficient planning and delivery of a tailored rehabilitation programme. Sporadic cross-referral alone is inadequate. The overarching objective is restoration of the fullest possible lifestyle and range of activity, irrespective of age.

(xi) Ensure that goal setting meetings during stroke rehabilitation are timetabled into the working week

(xii) Involve the person with stroke and, where appropriate, their family or carer in the discussion.

For younger people with stroke, crucial consideration of employment and career - sometimes inappropriately deferred should be built into the planning process from the outset.

\section{Return to work}

(xiii) Return to work issues should be identified as soon as possible after the person's stroke, reviewed regularly and managed actively. Active management should include:

$>$ identifying the physical, cognitive, communication and psychological demands of the job (for example, multitasking by answering emails and telephone calls in a busy office)

> identifying the impacts of any impairments on work performance (for example, physical limitations, anxiety, fatigue preventing attendance for a full day at work, cognitive impairments preventing multitasking and communication deficits)

$>$ tailoring an intervention (for example, teaching strategies to support multitasking or memory difficulties, teaching the use of voice activated software for people with difficulty typing, and delivery of work simulations)

> educating about the Equality Act 2010 and support available (for example, an access to work scheme)

> workplace visits and liaison with employers to establish reasonable accommodations, such as provision of equipment and graded return to work.

\section{Intensity of stroke rehabilitation}

(xiv) Offer initially at least 45 minutes of each relevant stroke rehabilitation therapy for a minimum of 5 days per week to those with the ability to participate, and where functional goals can be achieved. If more rehabilitation is needed at a later stage, tailor the intensity to the person's needs at that time.

\section{Swallowing}

(xv) Offer swallowing therapy at least 3 times a week to those with dysphagia able to participate, for as long as they continue to make functional gains. Swallowing therapy could include compensatory strategies, exercises and postural advice.

\section{Providing support and information}

(xvi) Provision of appropriate, accurate and timely information is a key component of post-stroke care.

> Working with the person with stroke and their family or carer, identify their information needs and how to deliver them, taking into account specific impairments such as aphasia and cognitive impairments. Pace the information to the person's emotional adjustment.

$>$ Review information needs at the person's 6-month and annual stroke reviews and at the start and completion of any intervention period.

\section{Long-term health and social support}

Although at some point after stroke, further resolution of neurological impairment may dwindle or cease, continuing reintegration into an individual's family, social and community life can still be enabled. Rehabilitation services should aim to 
withdraw only when the knowledge, skills and, if necessary, support are in place to achieve these aims.

(xvii) Inform people after stroke that they can self-refer, usually with the support of a GP or named contact, if they need further stroke rehabilitation services.

(xviii) Provide information so that people after stroke are able to recognise the development of complications of stroke, including frequent falls, spasticity, shoulder pain and incontinence.

(xix) Encourage people to focus on life after stroke and help them to achieve their goals. This may include:

> facilitating their participation in community activities, such as shopping, civic engagement, sports and leisure pursuits, visiting their place of worship and stroke support groups

> supporting their social roles, for example, work, education, volunteering, leisure, family and sexual relationships

$>$ providing information about transport and driving (including DVLA requirements; see www.dft.gov.uk/ dvla/medical/aag).

(xx) Review health and social care needs of people after stroke, and the needs of their carers, at 6 months and annually thereafter. These reviews should cover participation and community roles to ensure that people's goals are addressed.

\section{Implications for implementation - barriers and facilitators}

The recommended initial rehabilitation in a dedicated stroke unit still needs attention as only $83.5 \%$ of patients are cared for in stroke units for at least $90 \%$ of their stay. ${ }^{7}$

Conversely, the delivery of ongoing and longer term stroke rehabilitation varies unacceptably across the UK. Lines of accountability and continuity of care are unclear or suboptimal in some centres, particularly in the context of intercurrent health problems. Patients living with the long-term sequelae of stroke may be cared for in a variety of settings, including general medical and clinical gerontology units after intercurrent illness, and for their on-going needs in community-based rehabilitation services. Patients need to be able to access appropriate skilled rehabilitation at any point in their stroke journey. Given the variability of rehabilitation service provision nationally, a national rehabilitation strategy is needed.

There is a paucity of community-based psychology services and speech and language therapists to manage emotional distress, cognitive deficits and speech problems to the required intensity with an agreed assessment and management plan, in line with recommendation xiv above.

Current organisational and budgetary boundaries between hospital and community care and between health and social care result in disintegration inappropriate to the ongoing complex needs of people with stroke. There are avoidable delays in transfers of care, treatment and appropriate placement. The appointment of stroke rehabilitation professionals, including physicians and therapists, with joint responsibilities across secondary and community care should be encouraged, as well as the joint provision of health and social care within an integrated service.
Probably the greatest unmet challenge is in the domain of return to work. Approximately $30 \%$ of people with stroke are of working age, yet access to vocational rehabilitation services is limited. If stroke broadly reflects long-term neurological conditions, at most $10 \%$ of the vocational rehabilitation needs of this group are met, ${ }^{8}$ even though vocational rehabilitation appears effective. ${ }^{9}$

It is hoped that derivation of service delivery standards and audit criteria based on CG162 will drive change for the better.

\section{Acknowledgements}

Members of the Guideline Development Group were: Khalid Ali, Martin Bird, Robin Cant, Sandra Chambers, Louise Clark, Tamara Diaz, Avril Drummond until October 2012, Anne Foster until March 2013, Kathryn Head, Pamela Holmes, Helen E Hunter, Najma Khan-Bourne, Keith MacDermott, Rory O'Connor, E Diane Playford (Chair) and Sue Thelwell. Members of the technical team were: Lola Adedokun, Katharina Dworzynski, Elisabetta Fenu, Lina Gulhane, Kate Lovibond, Antonia Morga, Jonathan Nyong, Grammati Sari and Gill Ritchie (guideline lead).

\section{References}

1 National Audit Office. Progress in improving stroke care: report by the comptroller and auditor general. London: National Audit Office, 2010. Available online at www.nao.org.uk/publications/0910/stroke.aspx [Accessed 5 August 2015].

2 Stroke Unit Trialists' Collaboration. Organised inpatient (stroke unit) care for stroke. Cochrane Database Syst Rev 2007;(4):CD000197.

3 National Institute for Health and Care Excellence. Stroke rehabilitation: long-term rehabilitation after stroke. Clinical Guideline 162. London: NICE, 2013. Available online at www.nice.org.uk/guidance/ cg162 [Accessed 5 August 2015].

4 National Institute for Health and Clinical Excellence. Stroke: diagnosis and initial management of acute stroke and transient ischaemic attack (TIA). London: NICE, 2008. Clinical Guideline 68. Available online at www.nice.org.uk/guidance/cg68 [Accessed 5 August 2015].

5 Intercollegiate Stroke Working Party. National clinical guideline for stroke, 4th edition. London: RCP, 2012.

6 National Institute for Health and Clinical Excellence 2006. Nutrition support in adults: Oral nutrition support, enteral tube feeding and parenteral nutrition. Clinical Guideline 32. London: NICE, 2006. Available online at www.nice.org.uk/guidance/cg32 [Accessed 5 August 2015].

7 Royal College of Physicians, Clinical Effectiveness and Evaluation Unit on behalf of the Intercollegiate Stroke Working Party. Sentinel Stroke National Audit Programme (SSNAP). London: RCP, 2014. Available online at www.strokeaudit.org/Newspress/SSNAP-AcuteOrganisational-Audit-2014-Public-Repor.pdf [Accessed 2 September 2015].

8 Playford ED, Radford K, Burton C et al. Mapping vocational rehabilitation services for people with long term neurological conditions. Available online at www.ltnc.org.uk/download_files/final\%20 reports/April\%2012/2012/2012/Vocational\%20rehabilitation Final\%20report.pdf [Accessed 2 September 2015].

9 Kenyon P, Koshy P, Wills-Johnson N. A cost benefit analysis of vocational rehabilitation services provided by CRS Australia. Perth: The John Curtin Institute of Public Policy, 2005. Available online at http://espace.library.curtin.edu.au/R?func=dbin-jump-full\&local_ base $=$ gen01-era02\&object_id=117902 [Access 2 September 2015].

Address for correspondence: Dr D Playford, UCL Institute of Neurology, Queen Square, London WC1N 3BG, UK.

Email: d.playford@ucl.ac.uk 